\title{
Spin-orbit effects in GaAs quantum wells: Interplay between Rashba, Dresselhaus, and Zeeman interactions
}

\author{
Enrico Lipparini,* Manuel Barranco, Francesc Malet, and Martí Pi \\ Departament ECM, Facultat de Física, and $\mathrm{IN}^{2}$ UB, Universitat de Barcelona, Diagonal 647, 08028 Barcelona, Spain \\ Llorenç Serra \\ Departament de Física, Universitat de les Illes Balears, and Institut Mediterrani d'Estudis Avançats IMEDEA (CSIC-UIB), \\ E-07122 Palma de Mallorca, Spain
}

(Received 24 March 2006; revised manuscript received 12 May 2006; published 6 September 2006)

\begin{abstract}
The interplay between Rashba, Dresselhaus, and Zeeman interactions in a quantum well submitted to an external magnetic field is studied by means of an accurate analytical solution of the Hamiltonian, including electron-electron interactions in a sum-rule approach. This solution allows us to discuss the influence of the spin-orbit coupling on some relevant quantities that have been measured in inelastic light scattering and electron-spin resonance experiments on quantum wells. In particular, we have evaluated the spin-orbit contribution to the spin splitting of the Landau levels and to the splitting of charge- and spin-density excitations. We also discuss how the spin-orbit effects change if the applied magnetic field is tilted with respect to the direction perpendicular to the quantum well.
\end{abstract}

DOI: 10.1103/PhysRevB.74.115303

PACS number(s): 73.21.Fg, 73.22.Dj, 73.22.Lp

\section{INTRODUCTION}

The study of spin-orbit (SO) effects in semiconductor nanostructures has been the object of many experimental and theoretical investigations in the last few years, see e.g., Refs. 1-16 and references therein. In spite of this, the extraction from measurements of the effective spin-orbit coupling constant of both Dresselhaus ${ }^{17}$ and Bychkov-Rashba ${ }^{18,19}$ SO interactions is not a simple matter, since the SO corrections to the electron-energy spectrum in a magnetic field $(B)$ are vanishingly small because they correspond to second-order effects in perturbation theory. Thus, few physical observables are sensitive enough to the SO interactions and allow for a quantitative estimate of their coupling constants. One such observable is the splitting of the cyclotron resonance (CR), which has been determined in transmission experiments with far-infrared radiation, ${ }^{20}$ and is due to the coupling between charge-density and spin-density excitations. ${ }^{21} \mathrm{~A}$ less clear example is the change in the Larmor frequency-spin splitting. ${ }^{22}$ The spin splitting has been observed in electron-spin resonance 23,24 and in inelastic light-scattering experiments. ${ }^{25,26}$

In this work we extend our previous results ${ }^{21,22}$ by obtaining an approximate, yet very accurate, analytical solution of the quantum-well SO Hamiltonian that contains both Dresselhaus and Bychkov-Rashba interactions. In the limit of high magnetic field, this solution coincides with the results of second-order perturbation theory, and allows us to study the SO corrections to the Landau levels in a simple way, and to study the transitions induced by an external electromagnetic field acting upon the system.

This work is organized as follows. In Sec. II we present the general formalism for the single-particle (sp) Hamiltonian. These results are used in Sec. III to study the transitions caused by an external electromagnetic field. The role of the electron-electron (e-e) interaction is discussed in Sec. IV within a sum-rule approach. In Sec. V we discuss the split- ting of the Landau levels and the appearance of charge- and spin-density modes making, whenever possible, qualitative comparisons with the experimental results. ${ }^{20,24,27-29}$ A brief summary is presented in Sec. VI, and the generalization of some of the expressions derived in Sec. II to the case of tilted magnetic fields is presented in the Appendix.

\section{SINGLE-PARTICLE STATES}

In the effective mass, dielectric-constant approximation, the quantum-well Hamiltonian $H$ can be written as $H=H_{0}$ $+\frac{e^{2}}{\epsilon} \sum_{i<j=1}^{N} \frac{1}{\left|\mathbf{r}_{i}-\mathbf{r}_{\mathbf{r}}\right|}$, where $H_{0}$ is the one-body Hamiltonian consisting of the kinetic, Zeeman, Rashba, and Dresselhaus terms,

$$
\begin{aligned}
H_{0} \equiv & \sum_{j=1}^{N}\left[h_{0}\right]_{j}=\sum_{j=1}^{N}\left[\frac{P^{+} P^{-}+P^{-} P^{+}}{4 m}+\frac{1}{2} g^{*} \mu_{B} B \sigma_{z}\right. \\
& \left.+\frac{\lambda_{R}}{2 i \hbar}\left(P^{+} \sigma_{-}-P^{-} \sigma_{+}\right)+\frac{\lambda_{D}}{2 \hbar}\left(P^{+} \sigma_{+}+P^{-} \sigma_{-}\right)\right]_{j} .
\end{aligned}
$$

$m=m^{*} m_{e}$ is the effective electron mass in units of the bareelectron mass $m_{e}, P^{ \pm}=P_{x} \pm i P_{y}, \sigma_{ \pm}=\sigma_{x} \pm i \sigma_{y}$, where the $\sigma$ 's are the Pauli matrices, and $\mathbf{P}=-i \hbar \boldsymbol{\nabla}+\frac{e}{c} \mathbf{A}$ represents the canonical momentum in terms of the vector potential $\mathbf{A}$, which in the following we write in the Landau gauge, $\mathbf{A}$ $=B(0, x, 0)$, with $\mathbf{B}=\boldsymbol{\nabla} \times \mathbf{A}=B \hat{\mathbf{z}}$. The second term in Eq. (1) is the Zeeman energy, where $\mu_{B}=\hbar e /\left(2 m_{e} c\right)$ is the Bohr magneton, and $g^{*}$ is the effective gyromagnetic factor. The third and fourth terms are the usual Rashba and Dresselhaus interactions, respectively. Note that for bulk GaAs, taken here as an example, $g^{*}=-0.44, m^{*}=0.067$, and the dielectric constant is $\epsilon=12.4$. To simplify the expressions, in the following we will use effective atomic units $\hbar=e^{2} / \epsilon=m=1$.

Introducing the operators 


$$
a^{ \pm}=\frac{1}{\sqrt{2 \omega_{c}}} P^{ \pm}
$$

with $\left[a^{-}, a^{+}\right]=1$ and $\omega_{c}=e B / c$ being the cyclotron frequency, the sp Hamiltonian $h_{0}$ can be rewritten as

$$
h_{0} / \omega_{c}=\frac{1}{2}\left(a^{+} a^{-}+a^{-} a^{+}\right)-\frac{1}{2} \frac{\omega_{L}}{\omega_{c}} \sigma_{z}-\frac{1}{2} i \tilde{\lambda_{R}}\left(a^{+} \sigma_{-}-a^{-} \sigma_{+}\right)+\frac{1}{2} \tilde{\lambda_{D}}\left(a^{+} \sigma_{+}+a^{-} \sigma_{-}\right),
$$

where $\omega_{L}=\left|g^{*} \mu_{B} B\right|$ is the Larmor frequency and $\tilde{\lambda}_{R, D}=\lambda_{R, D} \sqrt{\frac{2}{\omega_{c}}}$. For the spinor $|\phi\rangle \equiv\left(\begin{array}{c}\phi_{1} \\ \phi_{2}\end{array}\right)$ (we shall use " 1 " for the top component and " 2 " for the bottom component of any spinor), the Schrödinger equation $h_{0}|\phi\rangle=\varepsilon|\phi\rangle$ adopts the form

$$
\left[\begin{array}{cc}
\frac{1}{2}\left(a^{+} a^{-}+a^{-} a^{+}\right)-\omega_{L} /\left(2 \omega_{c}\right)-\varepsilon & i \tilde{\lambda_{R}} a^{-}+\tilde{\lambda_{D}} a^{+} \\
-i \tilde{\lambda_{R}} a^{+}+\tilde{\lambda_{D}} a^{-} & \frac{1}{2}\left(a^{+} a^{-}+a^{-} a^{+}\right)+\omega_{L} /\left(2 \omega_{c}\right)-\varepsilon
\end{array}\right]\left(\begin{array}{l}
\phi_{1} \\
\phi_{2}
\end{array}\right)=0 .
$$

We expand $\phi_{1}$ and $\phi_{2}$ into oscillator states $|n\rangle$ as $\phi_{1}$ $=\sum_{n=0}^{\infty} a_{n}|n\rangle, \phi_{2}=\sum_{n=0}^{\infty} b_{n}|n\rangle$, on which $a^{+}$and $a^{-}$act in the usual way, i.e., $\frac{1}{2}\left(a^{+} a^{-}+a^{-} a^{+}\right)|n\rangle=\left(n+\frac{1}{2}\right)|n\rangle, a^{+}|n\rangle=\sqrt{n+1} \mid n$ $+1\rangle, a^{-}|n\rangle=\sqrt{n}|n-1\rangle$, and $a^{-}|0\rangle=0$. This yields the infinite system of equations

$$
\begin{aligned}
& (n+\alpha-\varepsilon) b_{n}-i{\tilde{\lambda_{R}}}_{n} a_{n-1}+\tilde{\lambda}_{D} \sqrt{n+1} a_{n+1}=0, \\
& (n+\beta-\varepsilon) a_{n}+i{\tilde{\lambda_{R}}}^{n+1} b_{n+1}+\tilde{\lambda}_{D} \sqrt{n} b_{n-1}=0,
\end{aligned}
$$

for $n \geqslant 0$, with $a_{-1}=0, b_{-1}=0$, and $\alpha=\left(1+\omega_{L} / \omega_{c}\right) / 2, \beta=(1$ $\left.-\omega_{L} / \omega_{c}\right) / 2$.

\section{A. Case in which either $\lambda_{R}=0$ or $\lambda_{D}=0$}

When only the Rashba or Dresselhaus terms are considered, Eqs. (5) can be exactly solved. ${ }^{14,30-32}$ For the sake of completeness, we give here the corresponding results. In the $\lambda_{D}=0$ case, combining Eqs. (5) one obtains

$$
\begin{gathered}
{\left[(n+\alpha-\varepsilon)(n-1+\beta-\varepsilon)-n{\widetilde{\lambda_{R}^{2}}}^{2} b_{n}=0,\right.} \\
{\left[(n+\alpha-\varepsilon)(n-1+\beta-\varepsilon)-n \widetilde{\lambda}_{R}^{2}\right] a_{n-1}=0,}
\end{gathered}
$$

either of which yields the energies

$$
\varepsilon_{n}^{ \pm}=n \pm \sqrt{\frac{1}{4}\left(1+\frac{\omega_{L}}{\omega_{c}}\right)^{2}+\frac{2}{\omega_{c}} \lambda_{R}^{2} n}
$$

One also obtains

$$
\left(n-1+\beta-\varepsilon_{n}^{ \pm}\right) a_{n-1}^{\varepsilon_{n}^{ \pm}}=-i \tilde{\lambda_{R}} \sqrt{n} b_{n}^{\varepsilon_{n}^{ \pm}}
$$

which together with the normalization condition $\left|a_{n-1}^{\varepsilon_{n}^{ \pm}}\right|^{2}$ $+\left|b_{n}^{\varepsilon_{n}^{ \pm}}\right|^{2}=1$ exactly solves the problem [for $n=0, a_{-1}=0, b_{0}$ $=1$, and $\left.\varepsilon_{0}=\frac{1}{2}\left(1+\omega_{L} / \omega_{c}\right)\right]$.

Equations (6) indicate that in the series expansion of the spinor $|\phi\rangle$, only one $a_{i}$ and one $b_{i}$ coefficient appears. Specifically,

$$
\left|n_{d}\right\rangle=\left(\begin{array}{c}
a_{n-1}^{\varepsilon_{n}^{+}}|n-1\rangle \\
b_{n}^{\varepsilon_{n}^{+}}|n\rangle
\end{array}\right), \quad\left|n_{u}\right\rangle=\left(\begin{array}{c}
a_{n}^{\varepsilon_{n+1}^{-}}|n\rangle \\
b_{n+1}^{\varepsilon_{n+1}^{-}}|n+1\rangle
\end{array}\right)
$$

In the limit of zero spin-orbit, the spinors $\left|n_{d}\right\rangle$ and $\left|n_{u}\right\rangle$ become $|n\rangle\left(\begin{array}{l}0 \\ 1\end{array}\right)$ and $|n\rangle\left(\begin{array}{l}1 \\ 0\end{array}\right)$, respectively. The exact expressions for the $a_{i}$ and $b_{i}$ coefficients entering Eqs. (9) are easy to work out. Expressions valid up to $\lambda_{R, D}^{2}$ order are given in the next subsection.

The $\lambda_{R}=0$ case can be worked out similarly. One obtains the secular equation

$$
(n+\beta-\varepsilon)(n-1+\alpha-\varepsilon)-n{\widetilde{\lambda_{D}^{2}}}^{2} 0,
$$

which yields

$$
\varepsilon_{n}^{ \pm}=n \pm \sqrt{\frac{1}{4}\left(1-\frac{\omega_{L}}{\omega_{c}}\right)^{2}+\frac{2}{\omega_{c}} \lambda_{D}^{2} n} .
$$

One also obtains

$$
\left(n-1+\alpha-\varepsilon_{n}^{ \pm}\right) b_{n-1}^{\varepsilon_{n}^{ \pm}}=-\tilde{\lambda}_{D} \sqrt{n} a_{n}^{\varepsilon_{n}^{ \pm}},
$$

which together with the normalization condition $\left|a_{n}^{\varepsilon_{n}^{ \pm}}\right|^{2}$ $+\left|b_{n-1}^{\varepsilon_{n}^{ \pm}}\right|^{2}=1$ exactly solves the problem (in this case, for $n$ $=0, b_{-1}=0$, and $a_{0}=1$ ).

Again, in the series expansion of the spinor $|\phi\rangle$, only one $a_{i}$ and one $b_{i}$ coefficient appears;

$$
\left|n_{d}\right\rangle=\left(\begin{array}{c}
a_{n+1}^{\varepsilon_{n+1}^{-}}|n+1\rangle \\
b_{n}^{\varepsilon_{n+1}^{-}}|n\rangle
\end{array}\right), \quad\left|n_{u}\right\rangle=\left(\begin{array}{c}
a_{n}^{\varepsilon_{n}^{+}}|n\rangle \\
b_{n-1}^{\varepsilon_{n}^{+}}|n-1\rangle
\end{array}\right),
$$

and the same comments as before apply.

\section{B. General case when $\lambda_{R} \neq 0$ and $\lambda_{D} \neq 0$}

If both terms are simultaneously considered, the SO interaction couples the states of all Landau levels, and an exact analytical solution to Eqs. (5) is unknown, and likely does not exist. We are going to find an approximate solution that in the $\lambda_{R, D}^{2} / \omega_{c} \ll 1$ limit coincides with the results of secondorder perturbation theory, i.e., it is valid up to ${\widetilde{\lambda_{R, D}}}_{R}$ order, and 
it is quite accurate as compared with exact results obtained numerically. Combining Eqs. (5), one can write

$$
\begin{aligned}
& {\left[n+\alpha-\varepsilon-\tilde{\lambda}_{R}^{2} \frac{n}{n-1+\beta-\varepsilon}-\tilde{\lambda}_{D}^{2} \frac{n+1}{n+1+\beta-\varepsilon}\right] b_{n}=} \\
& -i \tilde{\lambda}_{R} \tilde{\lambda}_{D}\left[\frac{\sqrt{n(n-1)}}{n-1+\beta-\varepsilon} b_{n-2}-\frac{\sqrt{(n+1)(n+2)}}{n+1+\beta-\varepsilon} b_{n+2}\right]
\end{aligned}
$$

and

$$
\begin{aligned}
& {\left[n+\beta-\varepsilon-\widetilde{\lambda}_{R}^{2} \frac{n+1}{n+1+\alpha-\varepsilon}-\widetilde{\lambda}_{D}^{2} \frac{n}{n-1+\alpha-\varepsilon}\right] a_{n}=} \\
& \quad-i{\widetilde{\lambda_{R}}}_{\tilde{\lambda}_{D}}\left[\frac{\sqrt{n(n-1)}}{n-1+\alpha-\varepsilon} a_{n-2}-\frac{\sqrt{(n+1)(n+2)}}{n+1+\alpha-\varepsilon} a_{n+2}\right] .
\end{aligned}
$$

The approximate solution is obtained by taking $a_{n-2}=a_{n+2}$ $=b_{n-2}=b_{n+2}=0$ in the above equations. This means that for each level $|n\rangle$, the SO interaction is allowed to couple it only with the $|n-1\rangle$ and $|n+1\rangle$ levels. This solution, which consists of a $\left|n_{d}\right\rangle$ and a $\left|n_{u}\right\rangle$ spinor, is therefore obtained by solving first the secular, cubic equation

$$
\begin{gathered}
(n+\alpha-\varepsilon)(n-1+\beta-\varepsilon)(n+1+\beta-\varepsilon)={\widetilde{\lambda_{R}^{2}}}^{2} n(n+1+\beta \\
-\varepsilon)+\widetilde{\lambda}_{D}^{2}(n+1)(n-1+\beta-\varepsilon) .
\end{gathered}
$$

Together with the equations

$$
\begin{gathered}
(n-1+\beta-\varepsilon) a_{n-1}=-i \tilde{\lambda}_{R} \sqrt{n} b_{n}, \\
(n+1+\beta-\varepsilon) a_{n+1}=-\tilde{\lambda}_{D} \sqrt{n+1} b_{n},
\end{gathered}
$$

and the normalization condition $\left|a_{n-1}\right|^{2}+\left|a_{n+1}\right|^{2}+\left|b_{n}\right|^{2}=1$, they determine the $\left|n_{d}\right\rangle$ solution. The solution corresponding to the $\left|n_{u}\right\rangle$ spinor is obtained by solving the secular equation

$$
\begin{aligned}
& (n+\beta-\varepsilon)(n-1+\alpha-\varepsilon)(n+1+\alpha-\varepsilon)=\widetilde{\lambda}_{R}^{2}(n+1)(n-1 \\
& \quad+\alpha-\varepsilon)+{\widetilde{\lambda_{D}}}^{2} n(n+1+\alpha-\varepsilon) .
\end{aligned}
$$

Together with the equations

$$
\begin{gathered}
(n-1+\alpha-\varepsilon) b_{n-1}=-\tilde{\lambda_{D}} \sqrt{n} a_{n}, \\
(n+1+\alpha-\varepsilon) b_{n+1}=i \tilde{\lambda_{R}} \sqrt{n+1} a_{n},
\end{gathered}
$$

and $\left|a_{n}\right|^{2}+\left|b_{n-1}\right|^{2}+\left|b_{n+1}\right|^{2}=1$, they determine the $\left|n_{u}\right\rangle$ solution.
Since all the estimates available in the literature (see, for example, Refs. 15, 21, and 22, and references therein) yield $\lambda_{R, D}^{2}$ values of the order of $10 \mu \mathrm{eV}$, and $\omega_{c}$ in GaAs is of the order of the meV even at small $B(\sim 1 \mathrm{~T})$, it is worth examining the above solutions in the ${\widetilde{\lambda_{R, D}}}_{2}^{2}=2 \lambda_{R, D}^{2} / \omega_{c} \ll 1$ limit, in which the secular equations have solutions easy to interpret.

To order $\tilde{\lambda}_{R, D}^{2}$, the relevant solution to Eq. (16) containing both SO terms is

$$
\varepsilon_{n}^{d}=n+\alpha+2 n \frac{\lambda_{R}^{2}}{\omega_{c}+\omega_{L}}-2(n+1) \frac{\lambda_{D}^{2}}{\omega_{c}-\omega_{L}},
$$

which corresponds to the spinor $\left|n_{d}\right\rangle$,

$$
\left|n_{d}\right\rangle=\left(\begin{array}{c}
a_{n-1}^{\varepsilon_{n}^{d}}|n-1\rangle+a_{n+1}^{\varepsilon_{n}^{d}}|n+1\rangle \\
b_{n}^{\varepsilon_{n}^{d}}|n\rangle
\end{array}\right),
$$

with coefficients

$$
\begin{gathered}
a_{n-1}^{\varepsilon_{n}^{d}}=i \tilde{\lambda_{R}} \sqrt{n} \frac{\omega_{c}}{\omega_{c}+\omega_{L}}, \\
a_{n+1}^{\varepsilon_{n}^{d}}=-\tilde{\lambda_{D}} \sqrt{n+1} \frac{\omega_{c}}{\omega_{c}-\omega_{L}}, \\
b_{n}^{\varepsilon_{n}^{d}}=1-\frac{1}{2} \widetilde{\lambda}_{R}^{2} n\left(\frac{\omega_{c}}{\omega_{c}+\omega_{L}}\right)^{2}-\frac{1}{2} \widetilde{\lambda}_{D}^{2}(n+1)\left(\frac{\omega_{c}}{\omega_{c}-\omega_{L}}\right)^{2} .
\end{gathered}
$$

In the following, we will refer to this solution as the quasispin-down (qdown) solution, since in the zero-spin-orbit coupling limit $\left|n_{d}\right\rangle$ becomes $|n\rangle\left(\begin{array}{l}0 \\ 1\end{array}\right)$. Analogously, Eq. (18) has the solution

$$
\varepsilon_{n}^{u}=n+\beta-2(n+1) \frac{\lambda_{R}^{2}}{\omega_{c}+\omega_{L}}+2 n \frac{\lambda_{D}^{2}}{\omega_{c}-\omega_{L}},
$$

which corresponds to the spinor $\left|n_{u}\right\rangle$,

$$
\left|n_{u}\right\rangle=\left(\begin{array}{c}
a_{n}^{\varepsilon_{n}^{u}}|n\rangle \\
b_{n-1}^{\varepsilon_{n}^{u}}|n-1\rangle+b_{n+1}^{\varepsilon_{n}^{u}}|n+1\rangle
\end{array}\right),
$$

with coefficients

$$
\begin{gathered}
b_{n-1}^{\varepsilon_{n}^{u}}=\tilde{\lambda}_{D} \sqrt{n} \frac{\omega_{c}}{\omega_{c}-\omega_{L}}, \\
b_{n+1}^{\varepsilon_{n}^{u}}=i \tilde{\lambda}_{R} \sqrt{n+1} \frac{\omega_{c}}{\omega_{c}+\omega_{L}}, \\
a_{n}^{\varepsilon_{n}^{u}}=1-\frac{1}{2}{\widetilde{\lambda_{R}}}_{R}^{2}(n+1)\left(\frac{\omega_{c}}{\omega_{c}+\omega_{L}}\right)^{2}-\frac{1}{2} \widetilde{\lambda}_{D}^{2} n\left(\frac{\omega_{c}}{\omega_{c}-\omega_{L}}\right)^{2} .
\end{gathered}
$$

In the following, we will refer to this solution as the quasispin-up (qup) solution, since in the zero-spin-orbit coupling limit $\left|n_{u}\right\rangle$ becomes $|n\rangle\left(\begin{array}{l}1 \\ 0\end{array}\right)$. When either $\lambda_{R}$ or $\lambda_{D}$ are zero, Eqs. (21) and (24) reduce to the exact Eqs. (9) and (13), 
respectively, and the corresponding $a_{i}$ and $b_{i}$ coefficients, valid up to order $\lambda_{R, D}$, can be extracted from Eqs. (22) and (25). These equations show that $a_{n}$ and $b_{n}$ are of order $O(1)$, whereas $a_{n \pm 1}$ and $b_{n \pm 1}$ are of order $O\left(\lambda_{R, D}\right)$, and $a_{n \pm 2}$ and $b_{n \pm 2}$ are of order $O\left(\lambda_{R, D}^{2}\right)$. This shows that the neglected terms in Eqs. (14) and (15) are of order $O\left(\lambda_{R, D}^{4}\right)$.

The sp energies obtained from Eqs. (20) and (23), valid in the $\lambda_{R, D}^{2} / \omega_{c} \ll 1$ limit, are

$$
E_{n}^{d}=\left(n+\frac{1}{2}\right) \omega_{c}+\frac{\omega_{L}}{2}+2 n \lambda_{R}^{2} \frac{\omega_{c}}{\omega_{c}+\omega_{L}}-2(n+1) \lambda_{D}^{2} \frac{\omega_{c}}{\omega_{c}-\omega_{L}},
$$

$$
E_{n}^{u}=\left(n+\frac{1}{2}\right) \omega_{c}-\frac{\omega_{L}}{2}-2(n+1) \lambda_{R}^{2} \frac{\omega_{c}}{\omega_{c}+\omega_{L}}+2 n \lambda_{D}^{2} \frac{\omega_{c}}{\omega_{c}-\omega_{L}} .
$$

Together with the structure of the associated spinors, Eqs. (21) and (24), this sp energy spectrum constitutes one of the main results of our work. By suitable differences of these energies, one may obtain the sp transition energies discussed in the next section.

The above sp energies coincide with the ones that can be derived from second-order perturbation theory with the standard expression

$$
E_{n}^{(2)}=\frac{1}{4} \sum_{m \neq n} \frac{\left|\left\langle m\left|-i \tilde{\lambda}_{R} \omega_{c}\left(a^{+} \sigma_{-}-a^{-} \sigma_{+}\right)+\tilde{\lambda}_{D} \omega_{c}\left(a^{+} \sigma_{+}+a^{-} \sigma_{-}\right)\right| n\right\rangle\right|^{2}}{E_{n}^{0}-E_{m}^{0}},
$$

where $|n\rangle=|n, \uparrow\rangle,|n, \downarrow\rangle$ are the spin-up and spin-down eigenstates of the sp Hamiltonian $\frac{1}{2}\left(a^{+} a^{-}+a^{-} a^{+}\right) \omega_{c}-\frac{1}{2} \omega_{L} \sigma_{z}$ with eigenvalues $E_{n}^{0}(\uparrow)=\left(n+\frac{1}{2}\right) \omega_{c}-\frac{1}{2} \omega_{L}$ and $E_{n}^{0}(\downarrow)=(n$ $\left.+\frac{1}{2}\right) \omega_{c}+\frac{1}{2} \omega_{L}$, respectively.

The approximate solution [Eqs. (26)] is very accurate in the high $B$ limit (see below). It also carries interesting information in the opposite limit of vanishing $B$. In this limit $\left(\omega_{L}, \omega_{c} \ll \lambda_{R, D}^{2}\right)$, Eqs. (16) and (18) yield the solutions

$$
\begin{aligned}
& E_{n}^{d}=\sqrt{2 \omega_{c}\left[n \lambda_{R}^{2}+(n+1) \lambda_{D}^{2}\right]}, \\
& E_{n}^{u}=\sqrt{2 \omega_{c}\left[(n+1) \lambda_{R}^{2}+n \lambda_{D}^{2}\right]},
\end{aligned}
$$

which show that, at $B \simeq 0$, to order $\lambda_{R, D}^{2}$ the Landau levels are not split due to the SO interaction, as one might naively infer from Eqs. (26). Another merit of the approximate solution is that it displays in a transparent way the interplay between the three spin-dependent interactions, namely, Zeeman, Rashba, and Dresselhaus. Such interplay has also been discussed in Ref. 22, in relation with the violation of the Larmor theorem due to the SO couplings, and in Ref. 33, where the Zeeman and SO interplay is discussed using the unitarily transformed Hamiltonian technique. Note also that in GaAs quantum wells, which are the object of application in this paper, due to the sign of $g^{*}$, the lowest energy level is the qup one at the energy $E_{0}^{u}=\frac{1}{2} \omega_{c}-\frac{1}{2} \omega_{L}-2 \lambda_{R}^{2} \omega_{c} /\left(\omega_{c}+\omega_{L}\right)$, containing the Rashba contribution alone, whereas the following level is the qdown one at the energy $E_{0}^{d}=\frac{1}{2} \omega_{c}+\frac{1}{2} \omega_{L}-2 \lambda_{D}^{2} \omega_{c} /\left(\omega_{c}-\omega_{L}\right)$, containing the Dresselhaus contribution alone. For all the other levels both SO terms contribute to the level energies.

We have assessed the quality of the above analytical solutions, Eqs. (26), by comparing them with exact numerical results for some particular cases. Indeed, the exact solution to Eqs. (5) can be obtained in the truncated space spanned by the lower $\mathcal{N}$ oscillator levels. Mathematically, Eqs. (5) are then cast into a linear-eigenvalue problem of the type

$$
\mathbf{M}\left(\begin{array}{l}
\mathbf{a} \\
\mathbf{b}
\end{array}\right)=\varepsilon\left(\begin{array}{l}
\mathbf{a} \\
\mathbf{b}
\end{array}\right)
$$

where $\mathbf{M}$ is a $2 \mathcal{N} \times 2 \mathcal{N}$ matrix, while $\mathbf{a}$ and $\mathbf{b}$ are column vectors made with the sets of coefficients $\left\{a_{n}, n=0, \ldots, \mathcal{N}\right.$ $-1\}$ and $\left\{b_{n}, n=0, \ldots, \mathcal{N}-1\right\}$, respectively. We have diagonalized $\mathbf{M}$ using a large enough $\mathcal{N}$ to ensure good convergence in the lower eigenvalues. The top panel of Fig. 1 displays a comparison of numerical (symbols) and analytical (solid lines) energies as a function of the Rashba SO strength, for a fixed Dresselhaus strength, both in units of $\omega_{c}$, namely, $y_{R}=\lambda_{R}^{2} / \omega_{c}$ and $y_{D}=\lambda_{D}^{2} / \omega_{c}=0.01$. The chosen values for $y_{D}$ and $y_{R}$ are within the expected range for a GaAs quantum well. For instance, if $m \lambda_{R, D}^{2} / \hbar^{2} \sim 10 \mu \mathrm{eV}$ and $B \sim 1 \mathrm{~T}, \quad\left(m \lambda_{R, D}^{2} / \hbar^{2}\right) /\left(\hbar \omega_{c}\right) \sim 10^{-2}$. There is an excellent agreement between analytical and numerical results, differences starting to be visible only for strong Rashba intensities and high Landau bands. Actually, in Fig. 1 the largest value of the adimensional ratio between Rashba SO and cyclotron energy $y_{R}=\lambda_{R}^{2} / \omega_{c}$ is 0.05 , small enough to validate the analytical expression. Notice, however, that for larger $y_{R}$ values (not shown in the figure), i.e., for small enough $B$, Eqs. (26) no longer reproduce the numerical results. For GaAs this happens for magnetic fields below $0.1 \mathrm{~T}$. Similarly, the bottom panel of Fig. 1 displays a comparison of numerical (symbols) and analytical (solid lines) energies as a function of the Dresselhaus SO strength $y_{D}$, for a fixed Rashba strength $y_{R}=\lambda_{R}^{2} / \omega_{c}=0.01$. For every Landau level, both panels show a crossing between the $\left|n_{u}\right\rangle$ state, which is at lower energy for $y_{R, D} \ll 0.01$ because $g^{*}<0$, and the $\left|n_{d}\right\rangle$ state, which eventually lies lower in energy. This crossing is due to the interplay between both SO terms.

Figure 2 compares the $a_{n}$ and $b_{n}$ amplitudes from the numerical diagonalization with the analytical result of Eqs. (25). For this purpose, we have chosen the rightmost qup states of the third Landau band in both panels of Fig. 1. 

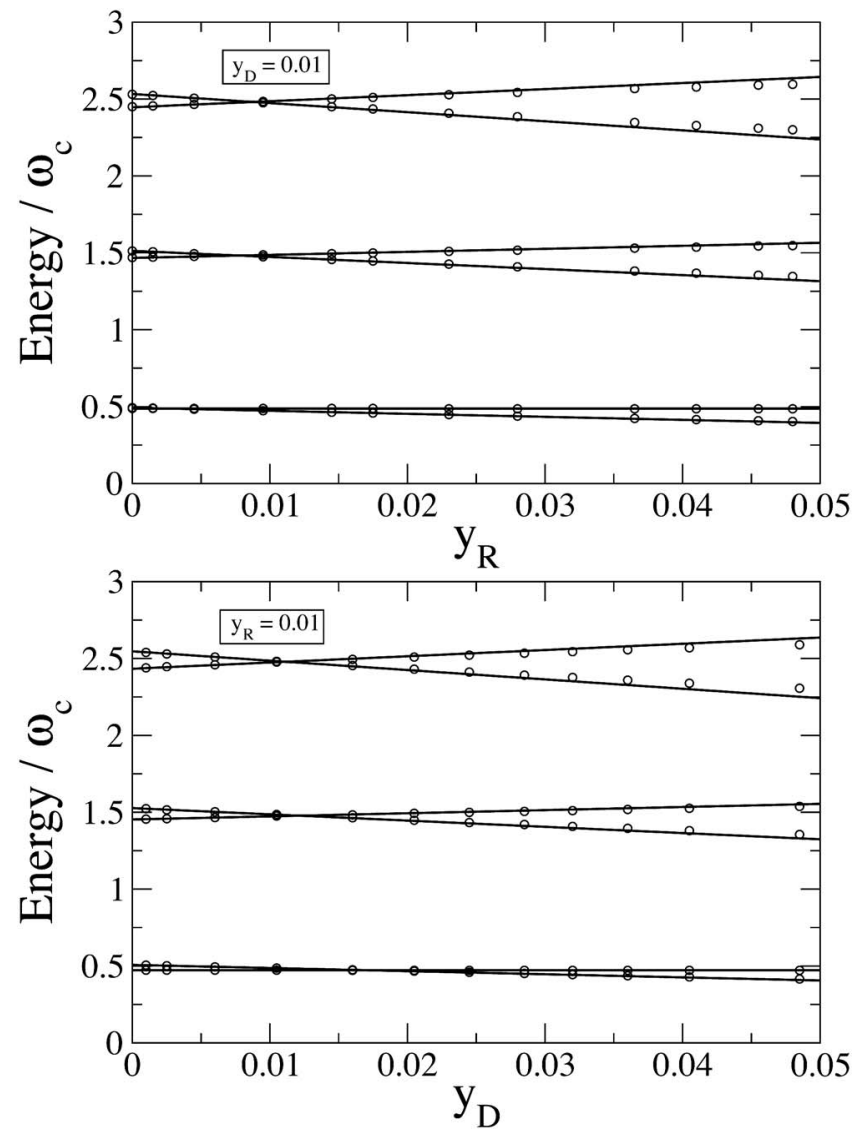

FIG. 1. Top panel: lower energy levels for a GaAs quantum well as a function of the Rashba intensity $y_{R}=\lambda_{R}^{2} / \omega_{c}$ for a fixed Dresselhaus intensity $y_{D}=\lambda_{D}^{2} / \omega_{c}=0.01$. The solid lines are the analytical result, Eqs. (26), while the symbols correspond to the exact diagonalization, Eq. (29). Bottom panel: lower energy levels for a GaAs quantum well as a function of the Dresselhaus intensity $y_{D}$ $=\lambda_{D}^{2} / \omega_{c}$ for a fixed Rashba intensity $y_{R}=\lambda_{R}^{2} / \omega_{c}=0.01$. The solid lines are the analytical result, Eqs. (26), while the symbols correspond to the exact diagonalization, Eq. (29).

These are the states with the largest SO intensities in Fig. 1. Note that even for these largest SO couplings, the analytical prediction is still excellent since the $a_{2}$ numerical and analytical amplitudes are very close, and there are only small $a_{0}$ and $a_{4}$ numerical corrections. For the $b_{n}$ amplitudes the comparison is also quite good and there are no relevant numerical corrections for $n$ different from 1 and 3, as predicted by Eqs. (25). For the qdown states similar results are found.

\section{SINGLE-PARTICLE LEVEL TRANSITIONS INDUCED BY APPLIED ELECTROMAGNETIC FIELDS}

We can use the preceding results to study the sp transitions induced in the system by the interaction with a leftcircular-polarized electromagnetic wave propagating along the $z$ direction, i.e., perpendicular to the plane of motion of the electrons, whose vector potential is $\mathbf{A}(t)=2 A(\cos \theta \hat{i}$ $+\sin \theta \hat{j})$, with $\theta=\omega t-q z$. The sp-interaction Hamiltonian $\mathbf{J} \cdot \mathbf{A} / c+g^{*} \mu_{B} \mathbf{s} \cdot(\boldsymbol{\nabla} \times \mathbf{A})$, where $\mathbf{J}=e \mathbf{v} / \sqrt{\boldsymbol{\epsilon}}$ reads
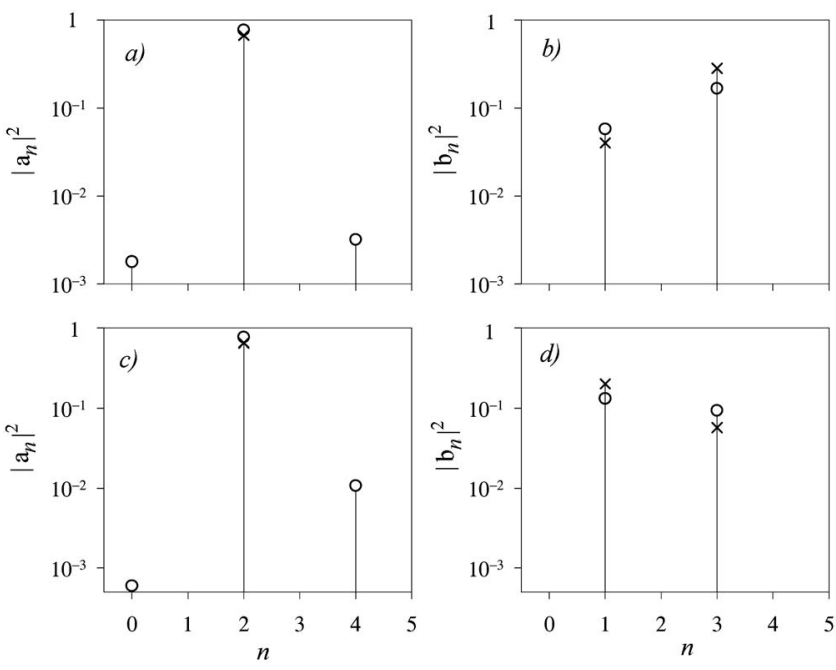

FIG. 2. Histograms with the $a_{n}$ and $b_{n}$ amplitudes for the rightmost qup state of the third Landau band of Fig. 1. Panels (a) and (b) are for $y_{R}=0.05$ and $y_{D}=0.01$, respectively, while (c) and (d) correspond to $y_{R}=0.01$ and $y_{D}=0.05$, respectively. The circles and crosses correspond to numerical and analytical results, i.e., to Eq. (29) and Eqs. (25), respectively.

$$
h_{i n t}=\frac{e}{c \sqrt{\epsilon}} A\left(v_{-} e^{i \theta}+v_{+} e^{-i \theta}\right)+\frac{1}{2} g^{*} \mu_{B} q A\left(\sigma_{-} e^{i \theta}+\sigma_{+} e^{-i \theta}\right) \text {, }
$$

where the velocity operator $v_{ \pm}$is defined as $v_{ \pm} \equiv$ $-i[x \pm i y, H]=P^{ \pm} \pm i \lambda_{R} \sigma_{ \pm}+\lambda_{D} \sigma_{\mp}$.

The Hamiltonian $h_{\text {int }}$ can be rewritten as

$h_{\text {int }}=\frac{e}{c \sqrt{\epsilon}} A \sqrt{2 \omega_{c}}\left(\alpha^{-} e^{i \theta}+\alpha^{+} e^{-i \theta}\right)+\frac{1}{2} g^{*} \mu_{B} q A\left(\sigma_{-} e^{i \theta}+\sigma_{+} e^{-i \theta}\right)$,

where the operators $\alpha^{+}$and $\alpha^{-}$acting on the spinor $|\phi\rangle$ are

$$
\alpha^{+}=\left[\begin{array}{cc}
a^{+} & i \tilde{\lambda_{R}} \\
\tilde{\lambda_{D}} & a^{+}
\end{array}\right], \quad \alpha^{-}=\left[\begin{array}{cc}
a^{-} & \tilde{\lambda_{D}} \\
-i \tilde{\lambda_{R}} & a^{-}
\end{array}\right] .
$$

In the dipole approximation $(q \approx 0)$, the charge-densityexcitation operator is $v_{ \pm}$. We note that, even in the presence of e-e interactions, this operator satisfies the f-sum rule,

$$
\begin{aligned}
\sum_{n}\langle 0|x \mp i y| n\rangle\left\langle n\left|-i v_{ \pm}\right| 0\right\rangle \\
\quad=\sum_{n} \omega_{n 0}|\langle n|x \pm i y| 0\rangle|^{2} \\
\quad=\frac{1}{2}\langle 0|[x \mp i y,[H, x \pm i y]]| 0\rangle=2 N,
\end{aligned}
$$

where $N$ is the electron number and $\omega_{n 0}$ are the excitation energies.

We consider next several useful examples of sp matrix elements involving the operators $\alpha^{+}$, which are proportional to $v_{+}$and $\sigma_{-}$, and the qup and qdown sp states of Eqs. (21) and (24). For the operator $\alpha^{+}$, we can write in general, 


$$
\left\langle\psi\left|\alpha^{+}\right| \phi\right\rangle=\psi_{1}^{*} a^{+} \phi_{1}+i \tilde{\lambda}_{R} \psi_{1}^{*} \phi_{2}+\tilde{\lambda}_{D} \psi_{2}^{*} \phi_{1}+\psi_{2}^{*} a^{+} \phi_{2},
$$

and have to distinguish between qup-qup, qdown-qdown, qup-qdown, and qdown-qup transitions. The qup-qup and qdown-qdown transitions represent the usual CR, and the qup-qdown and qdown-qup are related to spin-flip transitions.

Let us start with the qup-qup and qdown-qdown transitions. To the order $\lambda_{R, D}^{2}$ they are dominated by the transition $n \rightarrow n+1$ at the energies $E_{n+1}^{d}-E_{n}^{d}$ and $E_{n+1}^{u}-E_{n}^{u}$ with matrix elements $\left|\left\langle(n+1)_{u}\left|\alpha^{+}\right| n_{u}\right\rangle\right|=\left|\left\langle(n+1)_{d}\left|\alpha^{+}\right| n_{d}\right\rangle\right|=\sqrt{n+1}$. The energy splitting of the cyclotron resonance is

$$
\Delta E_{C R}=\left|4 \lambda_{R}^{2} \frac{\omega_{c}}{\omega_{c}+\omega_{L}}-4 \lambda_{D}^{2} \frac{\omega_{c}}{\omega_{c}-\omega_{L}}\right| .
$$

The $\alpha^{+}$excitation operator also induces a qup-qdown transition with energy $E_{n}^{d}-E_{n}^{u}$ and matrix element $\left|\left\langle n_{d}\left|\alpha^{+}\right| n_{u}\right\rangle\right|={\tilde{\lambda_{D}}}^{\omega_{L}} /\left(\omega_{c}-\omega_{L}\right)$. This is a spin-flip transition. In particular, when $n=0$ it is related to the Larmor resonance at the energy ${ }^{22}$

$$
\Delta E_{L}=\omega_{L}+2\left(\lambda_{R}^{2} \frac{\omega_{c}}{\omega_{c}+\omega_{L}}-\lambda_{D}^{2} \frac{\omega_{c}}{\omega_{c}-\omega_{L}}\right) .
$$

Note that the transition matrix element is linear in $\tilde{\lambda_{D}}$, and that in the presence of the Rashba interaction alone, $\alpha^{+}$ causes no spin-flip transition.

For the operator $\sigma_{-}$one gets $\left\langle\psi\left|\sigma_{-}\right| \phi\right\rangle=2 \psi_{2}^{*} \phi_{1}$. The dominant transition is the spin-flip excitation at energy $E_{n}^{d}-E_{n}^{u}$ with matrix element $\left|\left\langle n_{d}\left|\sigma_{-}\right| n_{u}\right\rangle\right|=2$. The qup-qup and qdown-qdown cyclotron resonances at energies $E_{n+1}^{d}-E_{n}^{d}$ and $E_{n+1}^{u}-E_{n}^{u}$ are also excited with strengths $\left|\left\langle(n+1)_{u}\left|\sigma_{-}\right| n_{u}\right\rangle\right|$ $=\left|\left\langle(n+1)_{d}\left|\sigma_{-}\right| n_{d}\right\rangle\right|=2 \tilde{\lambda_{D}} \sqrt{n+1} \omega_{c} /\left(\omega_{c}-\omega_{L}\right)$.

Other excitations that deserve some attention are those induced by the operators $\alpha^{+} \sigma_{ \pm}$and $\alpha^{+} \sigma_{z}$. They are detected in inelastic light-scattering experiments as spin-dipole resonances. ${ }^{27}$ The operator $\alpha^{+} \sigma_{z}$ excites the same cyclotron states as $\alpha^{+}$, at the energies $E_{n+1}^{d}-E_{n}^{d}$ and $E_{n+1}^{u}-E_{n}^{u}$, and with the same transition-matrix element $\sqrt{n+1}$. In contrast, the operator $\alpha^{+} \sigma_{+}$mainly induces the transition from qdown to qup states at the energy $E_{n+1}^{u}-E_{n}^{d}$, whereas the operator $\alpha^{+} \sigma_{-}$ induces the transition from qup to qdown states at the energy $E_{n+1}^{d}-E_{n}^{u}$. The transition-matrix elements are given by $K(n$ $\left.+1)_{u}\left|\alpha^{+} \sigma_{+}\right| n_{d}\right\rangle|=|\left\langle(n+1)_{d}\left|\alpha^{+} \sigma_{-}\right| n_{u}\right\rangle \mid=2 \sqrt{n+1}$. We thus see that the dipole transitions between Landau levels $|n\rangle$ and $\mid n$ $+1\rangle$ at "unperturbed" energies $E_{n+1}-E_{n}$ are split by the SO interaction, an effect that under some circumstances may be observed, as will be discussed in Sec. V.

\section{ELECTRON-ELECTRON INTERACTION AND SUM RULES}

In this section our aim is to discuss the role played by the e-e interaction in the physical processes in which $\mathrm{SO}$ effects can be important and, as a consequence, have a chance to be experimentally detected. Since we have obtained a spinor basis that includes the SO effects (SO basis), one might use it to diagonalize the Coulomb interaction. This has been done, for example, in Ref. 16, where the spinor basis [Eqs. (9)] has been used to study the influence of the Rashba interaction on the incompressible Laughlin state. One could also use the SO basis [Eqs. (21) and (24)] to solve the random-phase-approximation equations, ${ }^{34}$ or to study SO effects on the collective states of the quantum well in the adiabatic time-dependent local-spin-current density approximation. $^{22,35}$ We have chosen a different way to incorporate interaction effects that, while being more approximate, it is accurate enough and allows one to obtain simple analytical expressions for the quantities of interest here. It is the sum-rule approach, which is well suited to address the interplay between SO coupling and the e-e interaction in some relevant excitation processes.

Let us first recall that in the absence of the SO coupling, two important theorems hold for the quantum-well Hamiltonian $H$, in which the e-e interaction is included. They are the Kohn theorem

$$
\left[H, \sum_{j} P_{j}^{+}\right]=\omega_{c} \sum_{j} P_{j}^{+},
$$

which tells us that, in photoabsorption experiments on quantum wells, a narrow absorption peak must appear at the cyclotron frequency $\omega=\omega_{c}$ excited by the cyclotron operator $\Sigma_{j} P_{j}^{+}$, and the Larmor theorem

$$
\left[H, S_{-}\right]=\omega_{L} S_{-},
$$

which states that in inelastic light-scattering experiments at small transferred momentum, or in electron-spin resonance experiments, a narrow collective state must be excited by the Larmor operator $S_{-}=\Sigma_{j} \sigma_{-}^{j}$ at the Larmor frequency. These two modes are not influenced by the e-e interaction. Things radically change if we include in $H$ the SO interaction. We then obtain

$$
\left[H, \sum_{j} P_{j}^{+}\right]=\omega_{c} \sum_{j}\left(P^{+}+i \lambda_{R} \sigma_{+}+\lambda_{D} \sigma_{-}\right)_{j}
$$

and

$$
\left[H, S_{-}\right]=\omega_{L} S_{-}+\sum_{j}\left(2 i \lambda_{R} P^{-} \sigma_{z}+2 \lambda_{D} P^{+} \sigma_{z}\right)_{j},
$$

which show that the SO interaction couples the cyclotron (dipole and spin dipole) and Larmor modes. We have studied in Sec. III the effects of the SO coupling on these excitations in the absence of the Coulomb interaction. Now, we want to determine whether the presence of both the SO and Coulomb interactions has an effect on the Larmor and cyclotron frequencies or, on the contrary, the results of the previous section still hold. With this goal in mind, we introduce the following mixed-sum rules: ${ }^{36,37}$

$$
m_{k}^{ \pm}=\frac{1}{2} \sum_{n} \omega_{n 0}^{k}\left(\left\langle 0|F| \phi_{n}\right\rangle\left\langle\phi_{n}\left|G^{\dagger}\right| 0\right\rangle \pm\left\langle 0\left|G^{\dagger}\right| \phi_{n}\right\rangle\left\langle\phi_{n}|F| 0\right\rangle\right)
$$




$$
=\frac{1}{2}\left(\left\langle 0\left|F\left(H-E_{0}\right)^{k} G^{\dagger}\right| 0\right\rangle \pm\left\langle 0\left|G^{\dagger}\left(H-E_{0}\right)^{k} F\right| 0\right\rangle\right),
$$

where $|0\rangle$ and $\left|\phi_{n}\right\rangle$ are the exact ground states (g.s.) and excited states of the full Hamiltonian $H$ (including e-e interactions), and $\omega_{n 0}=E_{n}-E_{0}$ are the corresponding excitation energies. For $k=0-3$ we obtain

$$
\begin{aligned}
& m_{0}^{-}=\frac{1}{2}\left\langle 0\left|\left[F, G^{\dagger}\right]\right| 0\right\rangle, \\
& m_{1}^{+}=\frac{1}{2}\left\langle 0\left|\left[F,\left[H, G^{\dagger}\right]\right]\right| 0\right\rangle, \\
& m_{2}^{-}=\frac{1}{2}\left\langle 0\left|\left[[F, H],\left[H, G^{\dagger}\right]\right]\right| 0\right\rangle, \\
& m_{3}^{+}=\frac{1}{2}\left\langle 0\left|\left[[F, H],\left[H,\left[H, G^{\dagger}\right]\right]\right]\right| 0\right\rangle .
\end{aligned}
$$

Clearly, the more sum rules are known, the better the knowledge of the Hamiltonian spectrum. With the four sum rules of Eqs. (42) we can obtain information only on two excited states (see below). Consequently, we will limit the analysis to the cases in which either the Rashba or Dresselhaus SO terms are present because, as one can see from Eqs. (39) and (40) as well, in this case only two states would then be coupled by the corresponding SO interaction.

Let us first consider the case where $F=G=\sum_{i} P_{i}^{-}$, i.e., $G^{\dagger}$ is the cyclotron operator. Evaluating the commutators in Eqs. (42) we have, to order $\lambda_{R, D}^{2}$,

$$
\begin{gathered}
m_{0}^{-}=2 N \omega_{c}, \\
m_{1}^{+}=2 N \omega_{c}^{2}, \\
m_{2}^{-}=2 N \omega_{c}^{3}\left[1-\frac{2}{\omega_{c}}\left(\lambda_{R}^{2}-\lambda_{D}^{2}\right)\right], \\
m_{3}^{+}=2 N \omega_{c}^{4}\left[1-\frac{4}{\omega_{c}}\left(\lambda_{R}^{2}-\lambda_{D}^{2}\right)+\frac{2 \omega_{L}}{\omega_{c}^{2}}\left(\lambda_{R}^{2}+\lambda_{D}^{2}\right)\right] .
\end{gathered}
$$

To obtain these equations we have used that $\sum_{i} P_{i}^{-}|0\rangle=0$ and have assumed that the g.s. of the system is fully polarized, i.e., $\left\langle 0\left|\sum_{i} \sigma_{z}^{i}\right| 0\right\rangle=N$. As such, these expressions are useless unless the left-hand side can be directly evaluated from the definition Eq. (41), and this evaluation yields a closed expression for the excitation energies and transition matrix elements. This is the case if we consider either of the $\lambda_{R, D}$ terms alone, because only two states are excited by the cyclotron operator $G^{\dagger}=\sum_{i} P_{i}^{+}$acting on the g.s. $|0\rangle$. Dropping, e.g., the $\lambda_{R}$ term, a straightforward calculation yields

$$
\begin{gathered}
\pi_{1}=2 N \omega_{c}\left[1-\frac{2 \omega_{c}}{\left(\omega_{c}-\omega_{L}\right)^{2}} \lambda_{D}^{2}\right], \\
\pi_{2}=2 N \frac{2 \omega_{c}^{2}}{\left(\omega_{c}-\omega_{L}\right)^{2}} \lambda_{D}^{2},
\end{gathered}
$$

$$
\begin{aligned}
& \omega_{10}=\omega_{c}+\frac{2 \omega_{c}}{\omega_{c}-\omega_{L}} \lambda_{D}^{2}, \\
& \omega_{20}=\omega_{L}-\frac{2 \omega_{c}}{\omega_{c}-\omega_{L}} \lambda_{D}^{2},
\end{aligned}
$$

where $\pi_{1}$ and $\pi_{2}$ are the transition strengths to the cyclotron $\left|\phi_{n_{1}}\right\rangle$ and Larmor $\left|\phi_{n_{2}}\right\rangle$ states, $\pi_{1}=\left|\left\langle\phi_{n_{1}}\left|\Sigma_{i} P_{i}^{+}\right| 0\right\rangle\right|^{2}$ and $\pi_{2}$ $=\left|\left\langle\phi_{n_{2}}\left|\sum_{i} P_{i}^{+}\right| 0\right\rangle\right|^{2}$, and $\omega_{10}, \omega_{20}$ are the respective excitation energies. This is in full agreement with the results of Sec. III, and shows that the e-e interaction does not affect the frequency and transition strengths of the cyclotron and Larmor resonances.

The case $\lambda_{D}=0$ can be worked out similarly, and the same conclusion may be extracted. We recall and stress again the results obtained in the previous section, namely, that when $\lambda_{D}=0$, the Larmor state $\left|\phi_{n_{2}}\right\rangle$ is not excited by the cyclotron operator $\Sigma_{i} P_{i}^{+}\left(\pi_{2}\right.$ turns out to be zero $)$. Alternatively, all previous calculations could have been carried out using for $G^{\dagger}$ the Larmor operator, namely, $F=G=\Sigma_{i} \sigma_{+}^{i}$. Assuming again that $\left\langle 0\left|\sum_{i} \sigma_{z}^{i}\right| 0\right\rangle=N$, we obtain the same results and draw the same conclusions as before. This is a consequence of the structure of Eqs. (39) and (40).

Using more sum rules, e.g., $m_{4}^{-}$and $m_{5}^{+}$, one may obtain information on other states that can be excited by the cyclotron operator $\Sigma_{i} P_{i}^{+}$. Their consideration shows that the e-e interaction does not affect, to order $\lambda_{R, D}^{2}$, either the cyclotron or the Larmor state, whose frequencies are the same as determined in Sec. III when both the SO terms are included in $H$.

When the g.s. of the system has both qup and qdown occupied states, ${ }^{21}$ the spin-dipole operator $\sum_{i} P_{i}^{+} \sigma_{z}^{i}$ entering Eq. (40) excites a state at an energy $\omega_{c}(1+\mathcal{K})$ (see below), instead of $\omega_{c}$ as it corresponds to the cyclotron (chargedipole) operator $\Sigma_{i} P_{i}^{+}$, and the results in Eqs. (44) must be corrected for. This effect is not related to the SO interaction, and appears even in the absence of it. The spin-dipole operator does not commute with the e-e interaction as the cyclotron operator $\Sigma_{i} P_{i}^{+}$does, and $\mathcal{K}$ is precisely the contribution to the spin-dipole operator $m_{1}^{+}$sum rule, arising from the e-e interaction, when one takes $F=G=\Sigma_{i} P_{i}^{-} \sigma_{z}^{i}$ :

$$
\begin{aligned}
m_{1}^{+} & =\sum_{n} \omega_{n 0}\left|\left\langle\phi_{n}\left|\sum_{i} P_{i}^{+} \sigma_{z}^{i}\right| 0\right\rangle\right|^{2} \\
& =\frac{1}{2}\left\langle 0\left|\left[\sum_{j} P_{j}^{-} \sigma_{z}^{j},\left[H, \sum_{i} P_{i}^{+} \sigma_{z}^{i}\right]\right]\right| 0\right\rangle=N \omega_{c}^{2}(1+\mathcal{K}),
\end{aligned}
$$

where

$$
\mathcal{K}=\frac{1}{2 N \omega_{c}^{2}}\left\langle 0\left|\sum_{i<j} \nabla_{r_{i j}}^{2} V\left(r_{i j}\right)\left(\sigma_{z}^{i}-\sigma_{z}^{j}\right)^{2}\right| 0\right\rangle .
$$

$\mathcal{K}$ can be extracted from inelastic light-scattering experiments. ${ }^{27}$ It turns out to be zero for fully polarized ground states, and small and negative-of the order of $10^{-2}$-otherwise. Similarly, the spin-flip dipole operators $\Sigma_{i} P_{i}^{+} \sigma_{ \pm}^{i}$, whose excitations can be also measured by inelastic 
light scattering, do not commute with the e-e interaction, which give rise to some energy corrections. It turns out that these corrections are equal for the three-spin dipole operators $\Sigma_{i} P_{i}^{+} \sigma_{z, \pm}^{i}$ because the value of $\mathcal{K}$ is the same for all them. Hence, the energy splittings among these excitations are not influenced by the e-e interaction, depending only on the Zeeman and SO energies as found and discussed at the end of Sec. III.

Finally, we want to comment on the consequences of the failure of the Kohn theorem due to the SO coupling using the $m_{1}^{+}$sum rule for $F=\Sigma_{i} P_{i}^{-} \sigma_{z}^{i}$ and $G=\Sigma_{i} P_{i}^{-}$;

$$
\begin{aligned}
m_{1}^{+} & =\sum_{n} \omega_{n 0}\left\langle 0\left|\sum_{i} P_{i}^{-} \sigma_{z}^{i}\right| n\right\rangle\left\langle n\left|\sum_{j} P_{j}^{+}\right| 0\right\rangle \\
& =\frac{1}{2}\left\langle 0\left|\left[\sum_{i} P_{i}^{-} \sigma_{z}^{i},\left[H, \sum_{j} P_{j}^{+}\right]\right]\right| 0\right\rangle=\omega_{c}\left\langle 0\left|\sum_{i} \sigma_{z}^{i}\right| 0\right\rangle .
\end{aligned}
$$

This sum rule allows us to study the interplay between charge and spin modes. If we cast it into a sum over "spindipole states" $|m\rangle$ and another over "charge-dipole states" $|\rho\rangle$, we obtain

$$
\begin{aligned}
m_{1}^{+}= & \sum_{\rho} \omega_{\rho 0}\left\langle 0\left|\sum_{i} P_{i}^{-} \sigma_{z}^{i}\right| \rho\right\rangle\left\langle\rho\left|\sum_{j} P_{j}^{+}\right| 0\right\rangle \\
& +\sum_{m} \omega_{m 0}\left\langle 0\left|\sum_{i} P_{i}^{-} \sigma_{z}^{i}\right| m\right\rangle\left\langle m\left|\sum_{j} P_{j}^{+}\right| 0\right\rangle .
\end{aligned}
$$

If there is no SO coupling, Kohn's theorem holds, implying that $\left\langle m\left|\sum_{i} P_{i}^{+}\right| 0\right\rangle=0$. Thus, when the spin g.s. $2 S_{z}$ $=\left\langle 0\left|\sum_{i} \sigma_{z}^{i}\right| 0\right\rangle$ is not zero [otherwise, $m_{1}^{+}=0$ from Eq. (47)], only the density modes would contribute to $m_{1}^{+}$through the $\rho$ sum in Eq. (48). On the contrary, if the SO coupling is taken into account, Kohn's theorem is violated and the spin- and charge-dipole states are coupled to order $\lambda_{R, D}^{2}$, with $\left\langle m\left|\Sigma_{i} P_{i}^{+}\right| 0\right\rangle$ being now different from zero.

To be more quantitative, let us assume that only one charge-dipole state, the cyclotron state $|\rho\rangle$ at energy $E_{1}=\omega_{c}$ $+O\left(\lambda_{R, D}^{2}\right)$, contributes to the first sum in Eq. (48), and only one spin-dipole state $|m\rangle$, at energy $E_{2}=\omega_{c}(1+\mathcal{K})+O\left(\lambda_{R, D}^{2}\right)$, contributes to the second sum, where we have indicated by $O\left(\lambda_{R, D}^{2}\right)$ the SO correction to the cyclotron and spin-dipole energies. Let us define the mixed strengths

$$
\begin{aligned}
& \pi_{1}=\left\langle 0\left|\sum_{i} P_{i}^{-} \sigma_{z}^{i}\right| \rho\right\rangle\left\langle\rho\left|\sum_{j} P_{j}^{+}\right| 0\right\rangle, \\
& \pi_{2}=\left\langle 0\left|\sum_{i} P_{i}^{-} \sigma_{z}^{i}\right| m\right\rangle\left\langle m\left|\sum_{j} P_{j}^{+}\right| 0\right\rangle .
\end{aligned}
$$

Evaluating the sum rules $m_{0}^{-}$and $m_{1}^{+}$for the operators $G$ $=\Sigma_{i} P_{i}^{-}$and $F=\Sigma_{i} P_{i}^{-} \sigma_{z}^{i}$, one easily obtains

$$
\pi_{1}=\omega_{c}\left\langle 0\left|\sum_{i} \sigma_{z}^{i}\right| 0\right\rangle \frac{E_{2}-\omega_{c}}{E_{2}-E_{1}}=\omega_{c}\left\langle 0\left|\sum_{i} \sigma_{z}^{i}\right| 0\right\rangle\left[1-\frac{O\left(\lambda_{R, D}^{2}\right)}{\left|\omega_{c} \mathcal{K}\right|}\right],
$$

$$
\pi_{2}=\omega_{c}\left\langle 0\left|\sum_{i} \sigma_{z}^{i}\right| 0\right\rangle \frac{\omega_{c}-E_{1}}{E_{2}-E_{1}}=\omega_{c}\left\langle 0\left|\sum_{i} \sigma_{z}^{i}\right| 0\right\rangle \frac{O\left(\lambda_{R, D}^{2}\right)}{\left|\omega_{c} \mathcal{K}\right|} .
$$

Eqs. (50) explicitly show that if $\left\langle 0\left|\Sigma_{i} \sigma_{z}^{i}\right| 0\right\rangle=0$, or if the SO coupling is neglected, the mixed strength $\pi_{2}$ is zero, and the spin-dipole state cannot be excited in photoabsorption experiments. The strength $\pi_{2}$ is nonzero only at odd filling factors $\nu\left(\nu=2 \pi \ell^{2} n_{e}\right.$, where $\ell=\sqrt{\hbar c / e B}$ is the magnetic length and $n_{e}$ is the electron density), for which $2 S_{z} / N$ $=1 / \nu$. Besides, when the system is fully polarized at $\nu=1$, the operators $\Sigma_{i} P_{i}^{-}$and $\Sigma_{i} P_{i}^{-} \sigma_{z}^{i}$ coincide and excite the same mode, so there is no splitting. The SO corrections $O\left(\lambda^{2}\right)$ can be calculated by taking into account the occupation of the ground state, either using the sum rule approach of this section, or the method of unitarily transforming the Hamiltonian, as described in Refs. 21 and 33. This calculation yields the energy splitting of the CR we discuss in the next section.

\section{COMPARISON WITH EXPERIMENTS AND DISCUSSION}

An actual confrontation of the theoretical results we have obtained with the experiments is not an easy task because of the smallness of the SO effects, and because of the way they are presented in the available literature, which makes it extremely difficult to carry out a quantitative analysis of such a subtle effect. Thus, we have to satisfy ourselves with a semiquantitative analysis, or to point out that these results are compatible with fairly rough estimated values of the SO coupling constants. We present now three such examples and a possible way to increase SO effects so that they could be easier to determine.

Using unpolarized far-infrared radiation, Manger et al. ${ }^{20}$ have measured the cyclotron resonance in GaAs quantum wells at different electron densities. The main finding of the experiment is a well-resolved splitting of the CR for $\nu=3,5$, and 7, and no significant splitting for $\nu=1$ and for even filling factors. We have seen that the SO interaction couples charge-density and spin-density excitations yielding the SO splitting of the CR given in Eq. (35). However, this expression, by itself, is unable to explain the filling-factor dependence of the observed splitting, for which one has to bear in mind that the SO coupling between the $\Sigma_{i} P_{i}^{-}$and $\Sigma_{i} P_{i}^{-} \sigma_{z}^{i}$ operators is strongly enhanced when the spin g.s. is not zero, as explicitly shown in Eqs. (50). We have also noted that $\mathcal{K}$ contributes to the splitting. Equation (35) has to be generalized to include these features. We obtain

$$
\Delta E_{C R}=\left|\frac{2 S_{z}}{N} 4\left(\lambda_{R}^{2} \frac{\omega_{c}}{\omega_{c}+\omega_{L}}-\lambda_{D}^{2} \frac{\omega_{c}}{\omega_{c}-\omega_{L}}\right)+\mathcal{K} \omega_{c}\right|,
$$

where the factor $2 S_{z} / N$ takes into account the actual sp contents of the g.s. This equation, together with Eqs. (50), embodies the theoretical explanation of the experimental findings. ${ }^{20}$ In particular, it gives an appreciable splitting only 
for odd-filling factors, for which the spin ground state $S_{z}$ is not zero. The analysis of the experimental splittings using Eqs. (51) yields values for the quantity $m\left|\lambda_{R}^{2}-\lambda_{D}^{2}\right| / \hbar^{2}$ of about $30 \mu \mathrm{eV}$, in agreement with the ones recently used to reproduce the spin splitting in quantum dots ${ }^{15}$ and wells. ${ }^{22}$ This is, in our opinion, one of the most clear evidences of a crucial SO effect on a physical observable, because its absence would imply that the physical effect does not show up.

The spin splitting of the first three Landau levels of a GaAs quantum well has been measured in a magnetoresistivity experiment by Dobers et al. ${ }^{24}$ We have shown in the previous sections that this splitting is not influenced by the e-e interaction, and that there is no spin splitting as $B$ goes to zero [Eqs. (28)]. Both facts are in agreement with the analysis of the experimental data, and with previous theoretical considerations $^{38}$ about the $B$-dependence of the gyromagnetic factor $g^{*}$, whose determination was the physical motivation of the magnetoresistivity experiment presented in Ref. 24. These authors have derived a $B$ - and $n$-dependent $g^{*}$ factor $g^{*}(B, n)=g_{0}^{*}-c\left(n+\frac{1}{2}\right) B$, where $g_{0}^{*}$ and $c$ are fitting constants that depend on the actual quantum well. The possibility of a SO shift was not considered, and their chosen law for $g^{*}$ implies that the spin-splitting energy $\Delta E_{n}$ does depend on the Landau-level index $n$ entering in a $B^{2}$ term, as they have $\Delta E_{n}=\left|g^{*} \mu_{B} B\right|$. A $B$-dependence in $g^{*}$ is crucial to explain the experimental data, and also to reproduce them theoretically. ${ }^{22}$

For the spin splitting of the Landau levels we obtain

$$
\Delta E_{n}=\omega_{L}+2(2 n+1)\left(\lambda_{R}^{2} \frac{\omega_{c}}{\omega_{c}+\omega_{L}}-\lambda_{D}^{2} \frac{\omega_{c}}{\omega_{c}-\omega_{L}}\right)
$$

(recall that $\omega_{L}=\left|g^{*} \mu_{B} B\right|$ ), i.e., a splitting that increases with $n$ because of the SO coupling. This SO correction has been worked out for the $n=0$ level in Ref. 22 using the equationof-motion method. It is known that the experimental results ${ }^{24}$ for $n=1$ and 2 can be reproduced if $g^{*}$ depends on $n$ and $B$, as already shown in that reference. We have verified that the $n$-dependence of $g^{*}$ cannot be mimicked by the $n$-dependence introduced by the SO interaction, Eq. (52). Recently, the analysis of $g^{*}$ has been extended to a wider magnetic-field range using time-resolved Faraday rotation spectroscopy. ${ }^{28,29}$

As a third example, we address the inelastic lightscattering excitation of the spin-dipole modes at $\nu=2$ as measured in Ref. 27. For this filling factor, in the absence of SO coupling, the spin-density inter-Landau-level spectrum is expected to be a triplet mode ${ }^{34,39}$ excited by the three operators $\Sigma_{i} P_{i}^{+} \sigma_{z, \pm}^{i}$ with energy splittings given by the Zeeman energy $\omega_{L}$. In the presence of SO interactions, we still expect a triplet mode to appear. Indeed, for $\nu=2$ we have $S_{z}=0$ and the cyclotron and spin-dipole modes excited by the operator $\Sigma_{i} P_{i}^{+} \sigma_{z}^{i}$ are decoupled, as previously discussed. Thus for this operator only one single mode should be detected at an average energy $\omega=\omega_{c}(1+\mathcal{K})$. The other operators $\Sigma_{i} P_{i}^{+} \sigma_{ \pm}^{i}$ yield the two other spin-dipole modes at the energies

$$
E^{ \pm}=\omega_{c}(1+\mathcal{K}) \pm \omega_{L} \pm 4\left(\lambda_{R}^{2} \frac{\omega_{c}}{\omega_{c}+\omega_{L}}-\lambda_{D}^{2} \frac{\omega_{c}}{\omega_{c}-\omega_{L}}\right) .
$$

The splitting is thus symmetric and depends on the SO strengths. In the experiment, triplet excitations were ob- served in all measured samples up to electron densities corresponding to $r_{s}=3.3$ (we recall that $r_{s}=1 / \sqrt{\pi n_{e}}$ ). $B$ was accordingly changed to keep the filling factor at $\nu=2$. Only one triplet-mode spectrum at $B=2.2 \mathrm{~T}$ was shown. From this spectrum, we infer that there is space for a $\sim 5-10 \% \mathrm{SO}$ effect on the splitting, assuming that at this fairly small magnetic field, $g^{*}$ is that of bulk GaAs, $g^{*}=-0.44$. We have estimated that $m\left|\lambda_{R}^{2}-\lambda_{D}^{2}\right| / \hbar^{2} \simeq 10 \mu \mathrm{eV}$, in line with the previous findings. Systematic measurements, especially at high $B$ where the splitting is larger, are called for to allow for a more quantitative analysis.

Another unequivocal signature of spin-orbit effects in quantum wells would be the detection of the Larmor state in photoabsorption experiments. The strength of this transition is given by $2 \frac{\lambda_{D}^{2}}{\omega_{c}}\left(\frac{\omega_{L}}{\omega_{c}-\omega_{L}}\right)^{2}$ and only depends on the Dresselhaus SO coupling [see the comment immediately after Eq. (35)]. In most experiments, $B$ is perpendicularly applied to the plane of motion of the electrons, and for GaAs the strength is so small that it has never been resolved.

We finally discuss the effect of tilting the applied magnetic field using the expressions derived in the Appendix. Equation (A3) can be used to obtain the splitting of the cyclotron resonance, which generalizes Eq. (35) for tilted magnetic fields,

$$
\begin{aligned}
\Delta E_{C R}= & 4\left[\left(C_{R} \mathcal{V}+C_{D} \mathcal{Z}\right) \frac{1}{1+\left|g^{*}\right| m^{*} \mathcal{S} / 2}\right. \\
& \left.-\left(C_{R} \mathcal{Z}+C_{D} \mathcal{V}\right) \frac{1}{1-\left|g^{*}\right| m^{*} \mathcal{S} / 2}\right],
\end{aligned}
$$

where $C_{R, D} \equiv m \lambda_{R, D}^{2} / \hbar^{2}$, and the tilting angle $\theta$ enters the quantities $\mathcal{V}, \mathcal{Z}$, and $\mathcal{S}$ defined in the Appendix. Tilting effects might arise because of the $1-\left|g^{*}\right| m^{*} \mathcal{S} / 2$ denominator in the above equation, but sizable effects on $\Delta E_{C R}$ should only be expected for materials such that $\left|g^{*}\right| m^{*} / 2$ is large. This is not the case for GaAs, but it is, e.g., for InAs and InSb, which have $\left|g^{*}\right| m^{*} / 2=0.169$ and 0.355 , respectively. For the latter case the dependence of $\Delta E_{C R}$ with the in-of-well field $B_{x}$, with a fixed $B_{z}$, is shown in Fig. 3. Notice that $\Delta E_{C R}$ is sharply increased when $B_{x}$ exceeds a given value ( $1 \mathrm{~T}$ for the parameters in Fig. 3), which is proving the strong enhancement of SO effects introduced by the horizontal component of the tilted-field configuration. Figure 3 also shows the comparison with the exact diagonalization data (symbols), indicating that the analytical formula, Eq. (54), is accurate up to rather large tilting angles and for varying relative weights of Rashba and Dresselhaus terms. As a matter of fact, this analytical result does not depend on $B_{z}$ although, for the sake of comparison with the exact diagonalization, we have used $B_{z}=1 \mathrm{~T}$ in Fig. 3. The evolution with $B_{x}$ is not always monotonous, especially for $C_{R}>C_{D}$ where we find an initial decrease of $\Delta E_{C R}$ with increasing $B_{x}$, vanishing at $B_{x}$ $\sim 0.8 \mathrm{~T}$, and eventually increasing again.

We want to point out that the one-band effective mass approach (EMA) used here is most appropriate for wide-gap semiconductors like GaAs $\left(E_{g}=1.43 \mathrm{eV}\right)$, and it is currently used for spin-orbit studies in this material, see, e.g., Ref. 40 and references therein. For narrow-gap semiconductors like 


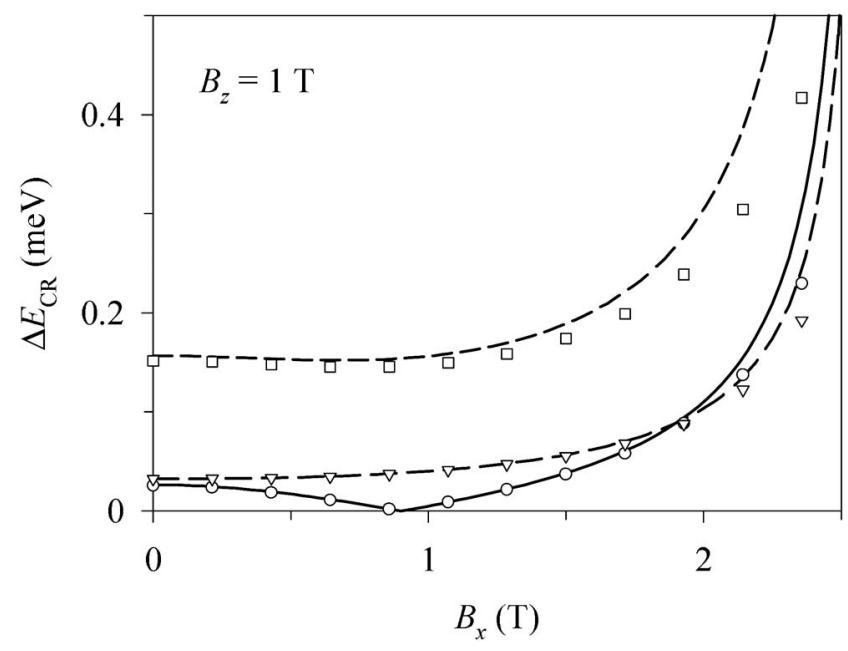

FIG. 3. Splitting of the cyclotron resonance for an InSb quantum well $\left(\left|g^{*}\right| m^{*} / 2=0.355\right)$ as a function of the in-of-well field $B_{x}$ when $B_{z}=1 \mathrm{~T}$. The lines are the result from the analytical formula, Eq. (54), while the symbols correspond to the exact diagonalization of Eq. (A1). Defining $C_{R, D}=m \lambda_{R, D}^{2} / \hbar^{2}$, the shown results are for: $C_{R}$ $=30 \mu \mathrm{eV}$ and $C_{D}=10 \mu \mathrm{eV}$ (solid line and circles); $C_{R}=10 \mu \mathrm{eV}$ and $C_{D}=10 \mu \mathrm{eV}$ (long-dashed line and triangles); $C_{R}=10 \mu \mathrm{eV}$ and $C_{D}=30 \mu \mathrm{eV}$ (short-dashed line and squares).

InSb $\left(E_{g}=0.18 \mathrm{eV}\right)$ still EMA can give accurate results if corrections coming from nonparabolicity are employed (as an alternative to multiband models). However, as far as no comparison with a given experiment is carried out for InSb (we basically look for physical phenomena), the obtained results, neglecting nonparabolicity, are still meaningful but of course more qualitative than for GaAs. It is also worth recalling that the envelope-function approximation implicit in EMA integrates the details described by the Bloch functions in the employed parameters (effective masses, Luttinger parameter for holes, etc. ${ }^{41}$ ) so that only the envelope functions remain, either in the presence or absence of an external magnetic field. We cannot disregard that for InSb the underlying band structure might have some influence on the optical transitions, but discussing it in detail is beyond the scope of our work. We also want to indicate that the largest employed magnetic field for $\operatorname{InSb}, B=2 \mathrm{~T}$, is still fairly small, so that the magnetic confinement radius is large and no relevant effects on a possible induced admixture of hole states is expected.

Finally, the tilting also affects the spin splitting of the Landau levels

$$
\begin{aligned}
\frac{\Delta E_{n}}{\omega_{c}}= & \frac{\left|g^{*}\right| m^{*}}{2} \mathcal{S}+2(2 n+1)\left[\left(y_{R} \mathcal{V}+y_{D} \mathcal{Z}\right) \frac{1}{1+\left|g^{*}\right| m^{*} \mathcal{S} / 2}\right. \\
& \left.-\left(y_{R} \mathcal{Z}+y_{D} \mathcal{V}\right) \frac{1}{1-\left|g^{*}\right| m^{*} \mathcal{S} / 2}\right],
\end{aligned}
$$

which generalizes Eq. (52) for $\theta \neq 0$. As we have commented before, in a recent experiment where spin-precession frequencies in a InGaAs quantum well have been measured using electrically detected electron-spin resonances, ${ }^{29}$ a strong dependence of the effective gyromagnetic factor $g^{e f f}$ on the applied tilted $B$ has been found. In particular, at $\theta$ $=45^{\circ}, g^{\text {eff }}$ exhibits oscillations with $B$, which indicate its sensitivity to the Landau-level filling, and a coupling between spin and orbital eigenstates, which is explicitly present in the spin-orbit term of Eq. (55). The effective $g$ factor that can be extracted from this equation at $\theta=45^{\circ}$, by taking the ratio $2 \Delta E_{n} /\left(m^{*} \mathcal{S} \omega_{c}\right)$, has the structure

$$
\left|g^{e f f}(B, n)\right|=\left|g_{0}^{*}\right|+\left(n+\frac{1}{2}\right)\left[c_{1} B+\frac{c_{2}}{B}\right],
$$

where the parametrization $g^{*}=g_{0}^{*}-c_{1}\left(n+\frac{1}{2}\right) B$ of Refs. 24 and 29 has been introduced in Eq. (55), and the $c_{2}$ term is the SO contribution. For the smaller $B$ values in the experiment, and for reasonable values of $m \lambda_{R, D}^{2} / \hbar^{2}$, of the order of $1-10 \mu \mathrm{eV}$, the SO contribution is important enough and should not be neglected; under these circumstances, timeresolved Faraday-rotation spectroscopy could be sensible to Rashba and/or Dresselhaus spin-orbit effects.

\section{SUMMARY}

We have discussed the appearance of spin-orbit effects in magnetoresistivity and inelastic light-scattering experiments on quantum wells. In particular, we have addressed SO effects on the splitting of the cyclotron resonance, on the sp Landau-level spectrum, and on spin-density excitations. Our discussion has been based on the use of an analytical solution of the quantum-well Hamiltonian valid up to second order in the SO coupling constants. The accuracy of this solution has been assessed comparing it with exact numerical diagonalizations.

We have carried out semiquantitative comparisons with available experimental data, with the twofold aim of extracting the value of the SO coupling constants and of indicating possible manifestations of the SO interactions. We have also pointed out that tilting the (usually) perpendicularly applied magnetic field might enhance spin-orbit effects, making them easier to detect.

\section{ACKNOWLEDGMENTS}

We would like to thank Josep Planelles for useful discussions. This work has been performed under Grants No. FIS2005-01414 and No. FIS2005-02796 from DGI (Spain) and Grant No. 2005 SGR00343 from Generalitat de Catalunya. E.L. has been supported by DGU (Spain), Grant No. SAB2004-0091.

\section{APPENDIX}

In this Appendix we generalize some of the expressions derived in Sec. II to the case in which $B$ has a in-of-well component, e.g., $B=\left(B_{x}, 0, B_{z}\right)$. The Zeeman term then becomes $\frac{1}{2} g^{*} \mu_{B} \mathbf{B} \cdot \boldsymbol{\sigma}=-\frac{1}{2} \omega_{L}^{z}\left(\sigma_{x} \tan \theta+\sigma_{z}\right)$, where we have introduced the zenithal angle $\theta, \tan \theta=B_{x} / B_{z}$, and the " $z$-Larmor" frequency $\omega_{L}^{z}=\left|g^{*} \mu_{B} B_{z}\right|$, with $\omega_{L}^{z} / \omega_{c}=\left|g^{*}\right| m^{*} / 2$. The Schrödinger [Eq. (4)] then becomes 


$$
\left[\begin{array}{cc}
\frac{1}{2}\left(a^{+} a^{-}+a^{-} a^{+}\right)-\omega_{L}^{z} /\left(2 \omega_{c}\right)-\varepsilon & i \tilde{\lambda}_{R} a^{-}+\tilde{\lambda}_{D} a^{+}-\left[\omega_{L}^{z} /\left(2 \omega_{c}\right)\right] \tan \theta \\
-i \tilde{\lambda_{R}} a^{+}+\tilde{\lambda_{D}} a^{-}-\left[\omega_{L}^{z} /\left(2 \omega_{c}\right)\right] \tan \theta & \frac{1}{2}\left(a^{+} a^{-}+a^{-} a^{+}\right)+\omega_{L}^{z} /\left(2 \omega_{c}\right)-\varepsilon
\end{array}\right]\left(\begin{array}{l}
\phi_{1} \\
\phi_{2}
\end{array}\right)=0 .
$$

The calculation proceeds as before, Eqs. (5) becoming

$$
\begin{aligned}
& (n+\alpha-\varepsilon) b_{n}-\frac{\alpha-\beta}{2} \tan \theta a_{n}-i \tilde{\lambda_{R}} \sqrt{n} a_{n-1}+\tilde{\lambda_{D}} \sqrt{n+1} a_{n+1}=0, \\
& (n+\beta-\varepsilon) a_{n}-\frac{\alpha-\beta}{2} \tan \theta b_{n}+i \tilde{\lambda_{R}} \sqrt{n+1} b_{n+1}+\tilde{\lambda_{D}} \sqrt{n} b_{n-1}=0,
\end{aligned}
$$

where $\alpha=\left(1+\omega_{L}^{z} / \omega_{c}\right) / 2$ and $\beta=\left(1-\omega_{L}^{z} / \omega_{c}\right) / 2$.

The sp spectrum [Eqs. (26)] becomes

$$
\begin{aligned}
& E_{n}^{d}=\left(n+\frac{1}{2}\right) \omega_{c}+\frac{\omega_{L}^{z}}{2} \mathcal{S}+2 n\left[\mathcal{U}\left(\lambda_{R}^{2}+\lambda_{D}^{2}\right)+\left(\lambda_{R}^{2} \mathcal{V}+\lambda_{D}^{2} \mathcal{Z}\right) \frac{\omega_{c}}{\omega_{c}+\omega_{L}^{z} \mathcal{S}}\right]-2(n+1)\left[\mathcal{U}\left(\lambda_{R}^{2}+\lambda_{D}^{2}\right)+\left(\lambda_{R}^{2} \mathcal{Z}+\lambda_{D}^{2} \mathcal{V}\right) \frac{\omega_{c}}{\omega_{c}-\omega_{L}^{z} \mathcal{S}}\right], \\
& E_{n}^{u}=\left(n+\frac{1}{2}\right) \omega_{c}-\frac{\omega_{L}^{z}}{2} \mathcal{S}+2 n\left[\mathcal{U}\left(\lambda_{R}^{2}+\lambda_{D}^{2}\right)+\left(\lambda_{R}^{2} \mathcal{Z}+\lambda_{D}^{2} \mathcal{V}\right) \frac{\omega_{c}}{\omega_{c}-\omega_{L}^{z} \mathcal{S}}\right]-2(n+1)\left[\mathcal{U}\left(\lambda_{R}^{2}+\lambda_{D}^{2}\right)+\left(\lambda_{R}^{2} \mathcal{V}+\lambda_{D}^{2} \mathcal{Z}\right) \frac{\omega_{c}}{\omega_{c}+\omega_{L}^{z} \mathcal{S}}\right],
\end{aligned}
$$

where we have defined $\mathcal{S}=1 / \cos \theta, \mathcal{U}=\sin ^{2} \theta / 4, \mathcal{V}=(1+\cos \theta)^{2} / 4$, and $\mathcal{Z}=(1-\cos \theta)^{2} / 4$. When $\theta=0, \mathcal{U}=\mathcal{Z}=0, \mathcal{V}=1$, and Eqs. (A3) reduce to Eqs. (26).

*Permanent address: Dipartimento di Fisica, Università di Trento, and INFN, 38050 Povo, Trento, Italy.

${ }^{1}$ C.-M. Hu, J. Nitta, T. Akazaki, H. Takayanagai, J. Osaka, P. Pfeffer, and W. Zawadzki, Phys. Rev. B 60, 7736 (1999).

${ }^{2}$ D. Richards and B. Jusserand, Phys. Rev. B 59, R2506 (1999).

${ }^{3}$ E. de Andrada e Silva, Phys. Rev. B 60, 8859 (1999).

${ }^{4}$ A. Voskoboynikov, S. S. Liu, C. P. Lee, and O. Tretyak, J. Appl. Phys. 87, 1 (2000).

${ }^{5}$ A. G. Mal'shukov and K. A. Chao, Phys. Rev. B 61, R2413 (2000).

${ }^{6}$ P. N. Racec, T. Stoica, C. Popescu, M. Lepsa, and T. G. van de Roer, Phys. Rev. B 56, 3595 (1997).

${ }^{7}$ O. Voskoboynikov, C. P. Lee, and O. Tretyak, Phys. Rev. B 63, 165306 (2001).

${ }^{8}$ J. A. Folk, S. R. Patel, K. M. Birnbaum, C. M. Marcus, C. I. Duruöz, and J. S. Harris, Jr., Phys. Rev. Lett. 86, 2102 (2001).

${ }^{9}$ B. I. Halperin, A. Stern, Y. Oreg, J. N. H. J. Cremers, J. A. Folk, and C. M. Marcus, Phys. Rev. Lett. 86, 2106 (2001).

${ }^{10}$ I. L. Aleiner and V. I. Fal'ko, Phys. Rev. Lett. 87, 256801 (2001).

${ }^{11}$ M. Valín-Rodríguez, A. Puente, Ll. Serra, and E. Lipparini, Phys. Rev. B 66, 165302 (2002).

${ }^{12}$ M. Valín-Rodríguez, A. Puente, and Ll. Serra, Phys. Rev. B 66, 045317 (2002).

${ }^{13}$ M. Valín-Rodríguez, A. Puente, Ll. Serra, and E. Lipparini, Phys. Rev. B 66, 235322 (2002).

${ }^{14}$ J. Schliemann, J. C. Egues, and D. Loss, Phys. Rev. B 67, 085302 (2003).

${ }^{15}$ J. Könemann, R. J. Haug, D. K. Maude, V. I. Fal'ko, and B. L. Altshuler, Phys. Rev. Lett. 94, 226404 (2005).
${ }^{16}$ M. Califano, T. Chakraborty, and P. Pietilainen, Phys. Rev. Lett. 94, 246801 (2005).

${ }^{17}$ G. Dresselhaus, Phys. Rev. 100, 580 (1955).

${ }^{18}$ Yu. A. Bychkov and E. I. Rashba, J. Phys. C 17, 6039 (1984).

${ }^{19}$ F. G. Pikus and G. E. Pikus, Phys. Rev. B 51, 16928 (1995).

${ }^{20}$ M. Manger, E. Batke, R. Hey, K. J. Friedland, K. Köhler, and P. Ganser, Phys. Rev. B 63, 121203(R) (2001).

${ }^{21}$ P. Tonello and E. Lipparini, Phys. Rev. B 70, 081201(R) (2004).

${ }^{22}$ F. Malet, E. Lipparini, M. Barranco, and M. Pi, Phys. Rev. B 73, 125302 (2006).

${ }^{23}$ D. Stein, K. v. Klitzing, and G. Weimann, Phys. Rev. Lett. 51, 130 (1982).

${ }^{24}$ M. Dobers, K. v. Klitzing, and G. Weimann, Phys. Rev. B 38, 5453 (1988).

${ }^{25}$ H. D. M. Davies, J. C. Harris, J. F. Ryan, and A. J. Turberfield, Phys. Rev. Lett. 78, 4095 (1997).

${ }^{26}$ M. Kang, A. Pinczuk, B. S. Dennis, M. A. Eriksson, L. N. Pfeiffer, and K. W. West, Phys. Rev. Lett. 84, 546 (2000).

${ }^{27}$ M. A. Eriksson, A. Pinczuk, B. S. Dennis, S. H. Simon, L. N. Pfeiffer, and K. W. West, Phys. Rev. Lett. 82, 2163 (1999).

${ }^{28}$ G. Salis, D. D. Awschalom, Y. Ohno, and H. Ohno, Phys. Rev. B 64, 195304 (2001).

${ }^{29}$ V. Sih, W. H. Lau, R. C. Myers, A. C. Gossard, M. E. Flatté, and D. D. Awschalom, Phys. Rev. B 70, 161313(R) (2004).

${ }^{30}$ E. I. Rashba, Fiz. Tverd. Tela (Leningrad) 2, 1224 (1960) [Sov. Phys. Solid State 2, 1109 (1960)].

${ }^{31}$ B. Das, S. Datta, and R. Reifenberger, Phys. Rev. B 41, 8278 (1990).

${ }^{32}$ V. I. Falko, Phys. Rev. B 46, 4320 (1992). 
${ }^{33}$ M. Valín-Rodríguez and R. G. Nazmitdinov, Phys. Rev. B 73, 235306 (2006).

${ }^{34}$ C. Kallin and B. I. Halperin, Phys. Rev. B 30, 5655 (1984).

${ }^{35}$ Ll. Serra, M. Barranco, A. Emperador, M. Pi, and E. Lipparini, Phys. Rev. B 59, 15290 (1999).

${ }^{36}$ E. Lipparini and S. Stringari, Phys. Rep. 175, 103 (1989).

${ }^{37}$ E. Lipparini, Modern Many Particle Physics-Atomic Gases, Quantum Dots and Quantum Fluids (World Scientific, Singapore, 2003).

${ }^{38}$ F. Malcher, G. Lommer, and U. Rössler, Superlattices Micro- struct. 2, 267 (1986); G. Lommer, F. Malcher, and U. Rössler, ibid. 2, 273 (1986).

${ }^{39}$ E. Lipparini, M. Barranco, A. Emperador, M. Pi, and Ll. Serra, Phys. Rev. B 60, 8734 (1999).

${ }^{40}$ P. Pietiläinen and T. Chakraborty, Phys. Rev. B 73, 155315 (2006).

${ }^{41}$ H. Haug and S. W. Koch, Quantum Theory of the Optical and Electronic Properties of Semiconductors, 4th ed. (World Scientific, Singapore, 2004). 